\title{
Estimation of Efforts During Testing of OOP using The AVISAR Framework
}

\author{
Prashant Vats, Manju Mandot
}

\begin{abstract}
In this research paper we have proposed our research work on the evaluation of efforts during the testing of OOP using the AVISAR object oriented testing framework which is based on the Genetic Algorithm. The proposed framework AVISAR provides a platform to address the issues which are related to the testing of Object Oriented aspects of OOP like Polymorphism, Inheritance, Polymorphism etc. which plays a key role in Effort Estimation of an Object Oriented Software.

Keywords: Genetic Algorithm (GA), Inheritance, Polymorphism, Object Oriented Programs (OOP), Object Oriented Software under Test (OOSUT)
\end{abstract}

\section{INTRODUCTION}

An object-oriented testing framework should be able to distinguish between dissimilar entities. During testing the test coverage criteria plays a vital rule for selecting the test and at what time to stop it. When estimating OOS effort, the control flow chart approach also does not seem to be helpful as an OOS abstraction. Throughout the production and testing of OOS, the central problem with the application of conventional metrics is that it cannot determine the SUT complexity using the control framework. In most OOS, the methods called in classes that use objects in a system under test are so small that it is almost impossible to resolve the number of decisions in the control flow chart when these OOPs are tested for a SUT. The use of the GA-based rolling test approach tackles the control flow accessibility problem in Object Oriented Software's flow path tests and helps the effective generation of Object Oriented test data. It also aids the test developer in achieving the methods call patterns between Classes during the object call by procedures between these Classes to evaluate these OOPs to find out that which abstractions in these classes are important to evaluate object-oriented the software. Generally, the use of any OO metric means a broad technique to assess the estimate of effort while testing classes in an object-oriented program. But in addition, there are more complexities involved in these metrics during object call patterns between call procedures to access the different classes of a system under test. So in this paper we have proposed A methodology based on the use

Revised Manuscript Received on April 15, 2020.

* Correspondence Author

Prashant Vats*, AIMACT, Banasthali Vidyapith, Rajasthan, India. Email: prashantvats12345@gmail.com

Manju Mandot, Professor, J. R. N. Rajasthan Vidyapith, Udaipur, Rajasthan, India. Email: Manju.mandot@gmail.com

(c) The Authors. Published by Blue Eyes Intelligence Engineering and Sciences Publication (BEIESP). This is an open access article under the CC BY-NC-ND license (http://creativecommons.org/licenses/by-nc-nd/4.0/)
Genetic Algorithm for the effort estimation during the Testing of OOP using the AVISAR frame work [17].

\section{NEED FOR EFFORT ESTIMATION IN OOP}

To ensure the quality of a product, a better understanding is provided by the "object oriented metric" (OOM) for Object-oriented Software under Testing (OOSUT). It offers the needed access to process efficiency to improve the quality of work done at the project level. Object Oriented Features like such as information hiding, encapsulation, inheritance, and the object abstraction approach lead to the requirement for a specialized metric applied to $\mathrm{OO}$ systems to estimate effort during the testing. A well-designed OO metric must be able to meet the requirement for an indication of the extent to which concealment was attained in an "OO design" (OOD) to make sure OOD quality during the OOP test.

\section{RELATED WORK IN OOT}

Hiroki T., et al. [1] utilized formal tests based on a Unit-class Petri color network to analyze the present behavior of objects. This approach is does not depend upon specific requirements, design methods, and languages. Jeff O., et al. [2] used peer-based tests at the level of class integration to resolve the problem CITO ("Class Level Integration Test") with edge weights taken from quantitative coupling. By automating the CITO problem, the capacity of developers has improved, but at the same time it cannot be applied to bigger systems is one of the limitations. R.B. Borie, et al. [3] used class-level Flow Graph-based tests to allow automatic test cases generation from properly stated classes. In it, they initiated the building of a simple flowchart that also aids generation of test cases and coverage analysis. However, alternatively, it may not adequately examine class behavior as it does not model the space of class state. Taratip S., et al. [4] used class-based cut-off tests to find test sequences to assuage the use of test pieces. Its key benefit is that it causes more failures to be detected in a much shorter time; however it cannot be scaled for big systems. Frankl, P., et al. [5]. employed the class-level ASTOOT technique (i.e. "a set of tools for OOT") to produce test cases that observe class state transitions and state values. In this technique, the test execution system can automatically verify the accuracy of the test cases with a test oracle. However, because test cases are randomly produced, analysis of test coverage is hard to carry out and the anticipated outcome of test cases can be specified on a "Boolean" value label. Dasiewicz, P., et al. [6] employed system-level event flow tests to prepare test cases that highlight the interaction between interrelated events.

blished By:

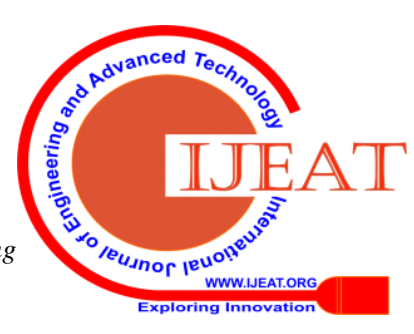

Blue Eyes Intelligence Engineering \& Sciences Publication (C) Copyright: All rights reserved. 
The detection of latent loops and restricts the loop path by manual intervention is its main advantage. Since it makes use of telephone "private branch exchange" (PBX) software, it is not appropriate for systems of small-scale. Parnas, D.L., et al. [7] employed class-based graph-based tests to replicate class behavior like a directed graph known as "the test graph". A test oracle can also be an application of this test graph. Manuel O., et al. [8] proposed a randomized Adaptive object-level test to assess the effectiveness of a test approach called "ARTOO" to discover actual flaws in actual software's. Wang, Y., et al. [9] to effectively state the behavior of classes or software modules, proposed "Trace specification rewrite" approaches for class-level testing. Provides canonical traits assigned directly between the crawl space and the class operation space. T.Y. Chen, et al. [10] has declared the state-based tests at the integrated class level to signify the changing states of the SUT. Use FSM to model an integrated system. Other test approaches proposed by the same authors [11] are tests based on peer-based events, which use the relationships between events to take advantage of the systems and verify the violation of restrictions. Tom Maibuam, et al. [12] employed tests based on the Integrated Class Level Coordination Contract to present a technique to implementing test cases with the Coordination Agreement concept. Through the use of contract, generation and execution of test cases can be automated. A. Jefferson, et al. [13] used integrated class-level category splitting techniques to demonstrate that present approach can discover flaws in OOS; the combination of the "category partition method" and a tool to detect memory management failures are extremely useful for OOT. They inspected a technique based on specifications with two small programs. Notkin, D., et al. [14] employed tests based on unit-level statistical algebraic abstractions, without requiring any specification, to automatically identify common and special unit tests for a class. It presents the portrayal of program behaviors and the detection of common and special tests through the use of statistical algebraic abstractions. M. Burrows, et al. [15] proposed another approach known as "Eraser" to dynamically detect data executions in multi-threaded block-based programs. It imposes a simple blocking discipline rather than seeking careers in general parallel programs. André B., et al. [16] proposed an algebra-based approach to the CSP process for class-level testing. Java is used for its implementation and it handles single processor and multiprocessor environments and also meets real-time priority programming requirements. For the object oriented paradigm, this technique simplifies the use of priorities.

\section{EFFORT ESTIMATION USING AVISAR}

The proposed OO metric tool in the OOT framework "AVISAR" consists mainly of three components: (i) Complexity calculator, (ii) Cohesion calculator, (iii) Estimator for OOD (OODE). With the use of these 3 components, it works collaboratively. The complexity degree involved during the various methods will be calculated by the Complexity calculator component, which will involve the class and individual weights assigning for these classes. The OODE will provide the measurement of the degree of characteristics such as encapsulation polymorphism, \& inheritance in an $\mathrm{OO}$ class. In addition, the cohesion component calculator provides the measurement of the extent of cohesion in a SUT. Using its all elements together to estimate the overall effort during testing and the development of an OOS. In this framework, for a given OO code block, the Lines of Code (LOC) number is fed to the input of a Requirement Model that lay out the NOC (number of classes) to forming the base for the estimation of the efforts in the proposed OOS. In addition, these NOCs are saved in requirements for the data dictionary storage that will provide as container for storing the requirement details for these NOCs produced for the provided code.

4.1 Cohesion Extent Calculator: As input, it accepts LOC. Using these LOC, a bipartite graph is formed. The functions and attribute called from a class in an OOS are used to make two nodes for the bipartite graph. A function of a class is linked to an attribute. If the attribute of this class is accessing this function, the degree of cohesion can be calculated as Max. $\{[$ Set of Functions*Set of Attributes $]$ - [Set of Bipartite Graph],0\}.

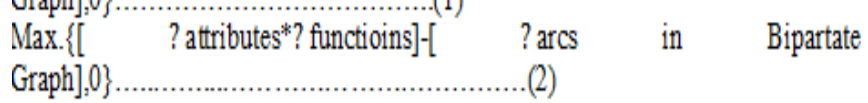

In AVISAR, during the repetitive process of generating the chromosome for the execution of the GA for an OOS, it requires an iterative process for the selection of one or more individual chromosomes. The execution of the test case selection occurs as explained below.

Let TS1 given as below be a chromosomal gene with 15 Test Cases that are chosen in a random order from the Initial population, provides a given test suite (TS1) which is as shown below.

\begin{tabular}{|c|c|c|c|c|c|c|c|c|c|}
\hline 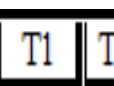 & \begin{tabular}{l|l}
$\mathrm{T} 2$ & $\mathrm{~T} 3$ \\
\end{tabular} & $\overline{1 T 4}$ & 155 & $\overline{76}$ & & 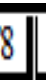 & & & $\overline{\mathrm{T} 1}$ \\
\hline \begin{tabular}{|l||}
$\mathrm{T}$ \\
\end{tabular} & \begin{tabular}{c|c|c|}
$T 12$ & $T 13$
\end{tabular} & $\overline{T 14}$ & T15 & 116 & 17 & & & 22 & $\mathrm{~T}$ \\
\hline 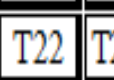 & \begin{tabular}{|l|l}
123 & $T 24$
\end{tabular} & $\overline{1725}$ & T26 & 227 & & & & & $4 \overline{T 32}$ \\
\hline \begin{tabular}{|l|l}
$\mathrm{T}$ \\
$\mathrm{T}$
\end{tabular} & \begin{tabular}{l|l}
134 & 735
\end{tabular} & & & & & & & & \\
\hline
\end{tabular}

The value of fitness function for the initial population of original Test Suite is given as below,

fvTS $=6.3532$.

The above value of fitness function for the initial population has been calculated by addition of the weights of the whole test cases that are being related to TS.

The value for the Fitness Functions of TS1, fvTS1 $=2.553$.

The initial probability for the crossover (Cp) of test gene is being chosen in a random manner and $\mathrm{Cp}=0.4$.

This value of the fitness function for a test gene is calculated by addition of the weights of whole set of test cases that are related to the chosen individual test genes during a genetic operation. The commencing test suite during the inception $\mathrm{TS} 1$, is as follows.

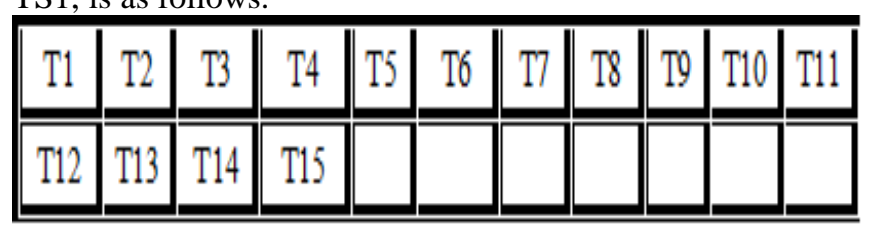

4.2 Complexity Calculator:
Published By:

Blue Eyes Intelligence Engineering \& Sciences Publication

(C) Copyright: All rights reserved.

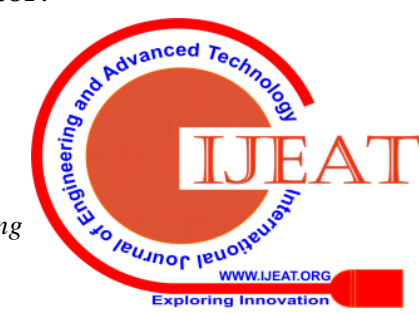


4.3 In AVISAR, during the OOD, it accepts the Number of Classes (NOC) as input to a OOSUT for calculating the level of complexity. Complexity can be calculated as when allocating weights to the $\mathrm{OO}$ classes:

Complexity level $\left.=\{\mathrm{l} / \mathrm{NOC})^{*} ?{ }_{\mathrm{i} 00}^{\mathrm{n}}\left(\mathrm{CW} \mathrm{n}(\mathrm{i}){ }^{*} \mathrm{NOM} \mathrm{n}(\mathrm{i})\right)\right\}$

Where,

$\mathrm{Cw} n(\mathrm{i})=$ Complexity w eights of the $\mathrm{n}$ Classes,

NOM $n(i)=$ Number of methods in individual $n$ classes.

While calculating the effort estimation in AVISAR, our aim is to keep the level of complexity for an OOSUT, as lower as possible. The consecutive iterations for generation of test suites in AVISAR are given as below. The reasonable predictive numbers are generated for each test case gene of TS1 are listed below in Table 1.1. where Mutation Probability $=0.10$

\begin{tabular}{|c|c|c|c|}
\hline \multicolumn{3}{|c|}{$R(T 1)$ for TS1 } \\
\hline \hline$T 1$ & 0.192 & $T 9$ & 0.333 \\
\hline$T 2$ & 0.458 & $T 10$ & 0.458 \\
\hline$T 3$ & 0.285 & $T 11$ & 0.088 \\
\hline$T 4$ & 0.235 & $T 12$ & 0.411 \\
\hline$T 5$ & 0.435 & $T 13$ & 0.723 \\
\hline$T 6$ & 0.788 & $T 14$ & 0.052 \\
\hline$T 7$ & 0.295 & $T 15$ & 0.482 \\
\hline$T 8$ & 0.794 & & \\
\hline
\end{tabular}

Table 1.1 Arbitrary numbers generated during the mutation with probability is 0.10 on TS1 for its each test case

During the successive iterations for generating the test suites using the GA, The Test Cases for the individual genes test sets are being generated in a random fashion with the mutation probability $(\mathrm{Mp}=0.10)$ were being restored by a set of new test genes from the native test suite (TS) that were not related to the TS1. The crucial tests T11 and T14 are substituted by new test cases from the initial population of the Test genes. These test genes are to be chosen in a random manner. The crucial tests T17 are being substituted with T16. These crucial tests cases are chosen in a random order, as they would be the next available new test genes from the actual lists of the crucial test gene set TS. Let us consider TS2 is to be the new test chromosomal gene that has been obtained as a result of the mutation and crossover operation on the test genes depending upon the value of their fitness function. The value of the fitness function of IP, fvTS is 6.3532 and value of the fitness function of TS2 which is given by fvTS2 is 2.4084 . After the first iteration in the test gene, the value of $\mathrm{Cp}$ is obtained as result of 2.4084 divide by 6.3532 , which is equal to 0.3791 , which is comparatively lesser than the value the initial crossover probability that is equal to 0.4 . So as a result the crossover operation first iteration in the test gene is being used as accepted convention and the new individual test gene is accepted as contemplate for the next iteration.
In AVISAR, the efficacy of this test suite has been evaluated and examines to present them separately by examining the other different metrics like, (i) Test Suite Efficiency, (ii) Complexity Calculation using APFD value, and (iii) Fault Coverage. As a resultant, the freshly resultant chromosome, TS2 is as shown below.

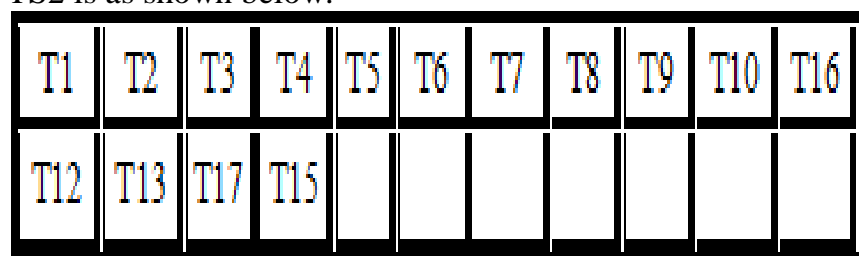

For the next successive alteration in the test chromosomal genes, the chosen individual test gene is TS2, which was generated as a resultant in the previous successive alteration in the test chromosomal genes. Let probability for the mutation of the successive alteration in the test chromosomal genes is 0.10 . The numbers generated randomly for each test chromosomal genes test cases are being shown as a list in the Table 1.2 given below.

\begin{tabular}{|c|c|c|c|}
\hline \multicolumn{4}{|c|}{ R(T1) for 'TS2 } \\
\hline \hline $\mathrm{T} 1$ & 0.002 & $\mathrm{~T} 9$ & 0.402 \\
\hline $\mathrm{T} 2$ & 0.399 & $\mathrm{~T} 10$ & 0.091 \\
\hline $\mathrm{T} 3$ & 0.351 & $\mathrm{~T} 163$ & 0.181 \\
\hline $\mathrm{T} 4$ & 0.813 & $\mathrm{~T} 12$ & 0.525 \\
\hline $\mathrm{T} 5$ & 0.667 & $\mathrm{~T} 13$ & 0.782 \\
\hline $\mathrm{T} 6$ & 0.298 & $\mathrm{~T} 17$ & 0.453 \\
\hline $\mathrm{T} 7$ & 0.270 & $\mathrm{~T} 15$ & 0.610 \\
\hline $\mathrm{T} 8$ & 0.184 & & \\
\hline
\end{tabular}

Table 1.2 Arbitrary numbers generated during the mutation with probability is 0.10 on TS2 for its each test case

The Test cases T10 \& T1 are being substituted with new test cases in the test chromosomal genes from its existing population by utilizing the random selection approach new test cases in the test chromosomal genes. These test cases are being substituted with T19 \& T18, which would be available next to from its actual population, TS. The new test chromosomal genes are given as TS3, which will be attained after substituting these test genes that will meet the expectations of the convincing value of the fitness function.

4.4 OOD Estimator (OODE): In AVISAR, the OODE provides a general estimate of the various characteristics of OOD, such as inheritance depth encapsulation, visibility of methods $(\mathrm{Vm})$ and degree of polymorphism for the $\mathrm{OO}$ classes. This OODE component in a SUT takes its NOC as input. Further we will examine its various subcomponents as given below:

Published By:

Blue Eyes Intelligence Engineering DOI: 10.35940/ijeat.D8003.049420

Journal Website: www.ijeat.org

\& Sciences Publication

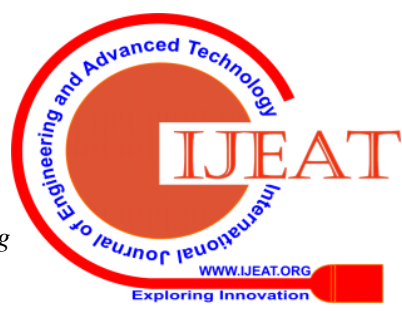


4.3.1 Visibility Calculator: The total no: of methods that would be visible in the given classes in a OOSUT will be calculated by this. Let us consider Tc be the complete no: of classes in the OOSUT, Let us say $\mathrm{Md}(\mathrm{Ci})$ be the no: of methods that are being asserted in a Class. If a class in OOSUT accesses a method by calling the object of that class, the predicate of class in OOSUT can be seen; otherwise, it cannot be seen, so the Visibility for a Method in the OOSSUT can be found as:

$$
\mathrm{Vm}=\sum_{\mathrm{j}=\mathrm{I}}^{\mathrm{Tc}} \frac{\text { Pradicate of }[\mathrm{Md}(\mathrm{Ci})] \text { i.e.Visible Classes }}{\mathrm{Tc}-1} .
$$

Where,

$\mathrm{Vm}=$ Visibility of Methods in a 00SUT's Class.

4.3.2 Encapsulation Measurement: The total number of classes Tc obtained from the NOC, in OOSUT is provided are feed as input to the Encapsulation Measurement component for OOSUT, provides the AHF and MHF for measuring the encapsulation for OOSUT, given as follows. The MHF and AHF jointly decide the encapsulation measurement of a class for OOSUT.

$\mathrm{AHF}=\sum_{\mathrm{i}=1}^{\mathrm{Tc}} \sum_{\mathrm{m}=1}^{\mathrm{Ad}}(1-\mathrm{Va}) / \sum_{\mathrm{i}=1}^{\mathrm{Tc}} \mathrm{Ad}$

Where,

$\mathrm{AHF}=$ Attribute Hiding factor

$\mathrm{Tc}=$ Total number of classes

$\mathrm{Ad}=$ Attributes declared in a class in a SUT

$\mathrm{Va}=$ Visibility of attributes by a class in a SUT .

$\mathrm{MHF}=\frac{\sum \mathrm{Tci}=1 \sum \mathrm{Mdm}=1(1-\mathrm{Vm})}{\sum \mathrm{Tci}=1 \mathrm{Md}}$

Where,

MHF = Methods Hiding factor,

$\mathrm{Tc}=$ Total number of classes,

$\mathrm{Md}=$ Number of methods declared in the class in a SUT,

$\mathrm{Vm}=$ Visibility of methods invoked by a class in a SUT.

4.3.3 Inheritance Measurement: In an OOSUT the inheritance in the $\mathrm{OO}$ classes can be obtained as two different measures for examining the level of inheritance. The total number of classes which is denoted by Tc, will be taken as input for an OOSUT. It mathematically determines the number of methods that can be referred within the interconnected class in an OOSUT as Ma.

$\mathrm{Ma}=\mathrm{Md}+\mathrm{Mi}$

Where,

$\mathrm{Ma}=$ Methods invoked in association with a Class.

$\mathrm{Md}=$ Methods declared in a Class

$\mathrm{Mi}=$ Methods inherited in a Class

So the Method associated component Method Inheritance Factor (MIF) would determine the Methods in OOSUT to be inherited as given below:

$\mathrm{MIF}=\frac{\sum \mathrm{Tci}=1 \mathrm{Mi}}{\sum \mathrm{Tci}=1 \mathrm{Ma}}$

Where,

MIF = Method Inheritance Factor,

For the Attribute Inheritance Factor (AIF), Attribute associated component would determine the number of attributes that can be approached in consortium with a $\mathrm{OO}$ class in a OOSUT as Aa.
$\mathrm{Aa}=\mathrm{Ad}+\mathrm{Ai}$

Where,

$\mathrm{Aa}=$ Number of attributes that can be accessed in association with class $\mathrm{i}$.

$\mathrm{Ad}=$ Number of attributes declared in a Class

$\mathrm{Ai}=$ Number of attributes from a base Class that are accessible to a Class $\mathrm{i}$ which is inherited from a base Class

So the Attribute Inheritance Factor (AIF) will determine the attributes that would be approachable to a Class I, that can be derived genetically from a parental OO Class shown as:

$\mathrm{AIF}=\frac{\sum \mathrm{TCi}=1 \mathrm{Ai}}{\sum \mathrm{TCi}=1 \mathrm{Ad}}$

Where,

AIF $=$ Attribute Inheritance Factor.

4.3.4 Polymorphism Factor: The "polymorphism factor" (PF) in an OOSUT is to ascertain the size of the possibility to apply the polymorphism. The implementing prospective for the polymorphism factor in an OOSUT can be estimated as: $\mathrm{PF}=\frac{\mathrm{Mo}}{\mathrm{Mn} * \mathrm{Dc}}$

Where,

$\mathrm{M}_{0}=$ Number of overriding methods in Class $\mathrm{i}$.

$\mathrm{Mn}=$ Number of new methods in Class $\mathrm{i}$.

$\mathrm{Dc}=$ Number of descendents of Class $\mathrm{i}$.

For calculating the Fitness value after the second successive alteration of IP, we have obtain the value of fvTS is to be equal to 6.3532.The fitness value of TS3 obtained during the second successive alteration of fvTS3 is to be equal to 2.3608 . After the third successive alteration the Current value of the factor Cp can be calculated by dividing the fitness value of TS3 by value of fvTS which is found to be equal to 0.3716 , which is less than the previous value of $\mathrm{Cp}$ which was equal to 0.3791 . so as a result both the mutation and crossover operations that were accomplished, are fully approved as a new chromosome which is given as below.

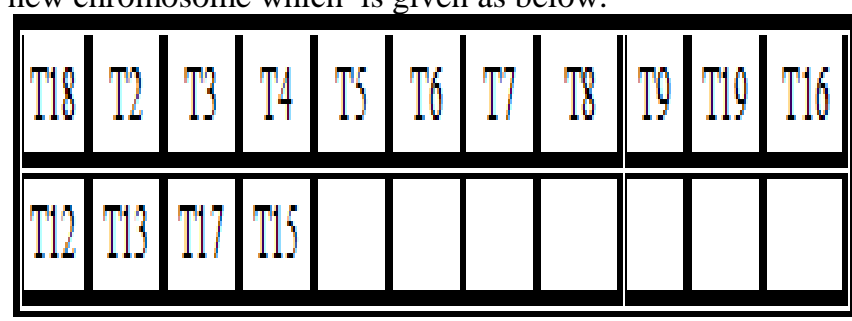

This chromosomal gene TS3 is contemplated for next successive alteration. The probability for the new mutation process is found to be equal to 0.60 . The successive random patterns of numbers are generated for the every test case of the chromosomal gene TS3 as shown below.

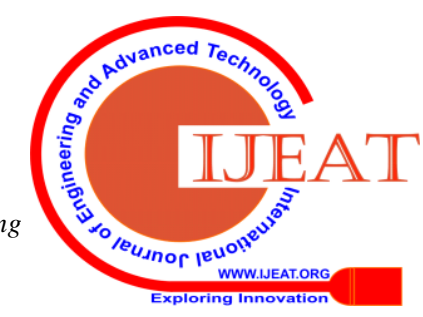




\begin{tabular}{|c|c|c|c|}
\hline \multicolumn{4}{|c|}{$R\left(T_{1}\right)$ for $T S 3$} \\
\hline $\mathrm{T} 18$ & 0.577 & $\mathrm{T9}$ & 0.644 \\
\hline $\mathrm{T} 2$ & 0.941 & T19 & 0.834 \\
\hline $\mathrm{T} 3$ & 0.001 & T16 & 0.537 \\
\hline $\mathrm{T} 4$ & 0.670 & $\mathrm{~T} 12$ & 0.802 \\
\hline T5 & 0.150 & T13 & 0.685 \\
\hline T6 & 0.295 & $\mathrm{~T} 17$ & 0.819 \\
\hline $\mathrm{T} 7$ & 0.165 & $\mathrm{~T} 15$ & 0.871 \\
\hline $\mathrm{T} 8$ & 0.964 & & \\
\hline
\end{tabular}

Table 1.3 Arbitrary numbers generated during the mutation with probability is 0.60 on TS3 for its each test case

During the third crossover the chromosomal test genes that tend to fail to meet the expected mutation probability were substituted. So these test cases T18, T3, T5, T6, T7, T16 are being substituted in accordance with the arbitrary test cases as T20, T21, T22,T23,T24, T25.

Suppose the new chromosomal test genes is to be TS4, which is a resultant of substitution of the chromosomal test genes. The Fitness value of IP is given be the fitness value (fvTS) which is found to be equal to 6.3532 and the fitness value (fvTS4) of the chromosomal test gene TS4 is found to be equal to 2.3283. After the third successive iteration, the value of Cp is obtained by dividing fvTS by fvTS4 and their value is found to be equal to 0.3665 , which is less than the fitness value of the TS3 which is 0.3716 . Therefore, after the successive genetic crossover and genetic mutation operations that are being on the Chromosomal test gene TS3 \& TS4 the new resultant chromosome TS4, is shown as follows.

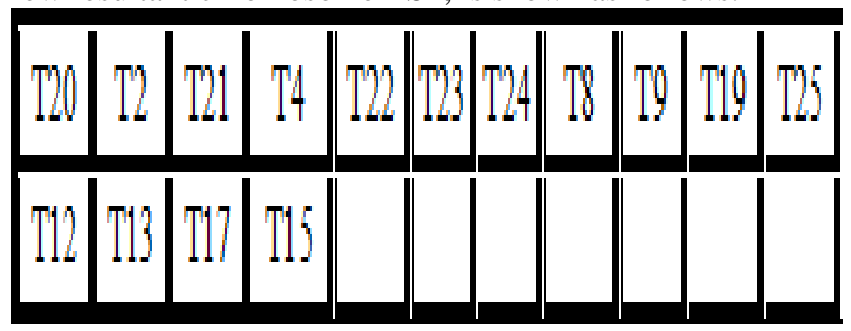

Let us further examine the Chromosomal test suite for the test gene TS4 for the next successive alteration. Here the probability of occurrence of the mutations on chromosomal test gene is fixed at the value equal to 0.50. During this successive alteration arbitrary numbers were generated during the mutation on the chromosomal test gene TS4 for its each test case.

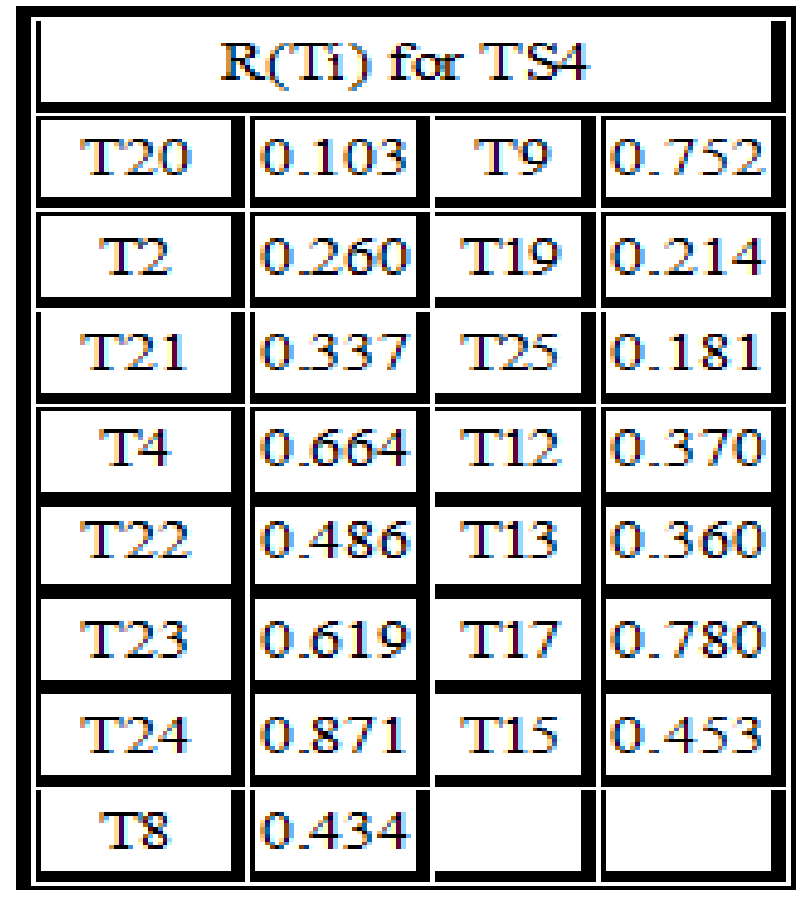

Table 1.4 Arbitrary numbers generated during the mutation with probability is 0.50 on TS4 for its each test case

During the fourth crossover the chromosomal test genes that tend to fail to meet the expected mutation probability were substituted. So these test cases T20, T2,T21, T22, T8, T19, T25, T12, T13, T15 are being substituted in accordance with the arbitrary test cases as T26, T27, T28,T29,T30, T31,T32, T33, T34, T35.

Suppose the new chromosomal test genes is to be TS5, which is a resultant of substitution of the chromosomal test genes. The Fitness value of IP is given be the fitness value (fvTS5) which is found to be equal to 6.3532 and the fitness value (fvTS5) of the chromosomal test gene TS5 is found to be equal to 2.9123. After the third successive iteration, the value of Cp is obtained by dividing fvTS by fvTS5 and their value is found to be equal to 0.4584 , which is not less than the fitness value of the TS3 which is 0.3716 . Therefore, after the successive genetic crossover and genetic mutation operations that are being on the Chromosomal test gene TS3 \& TS4 the new resultant chromosome TS5, is shown as follows.

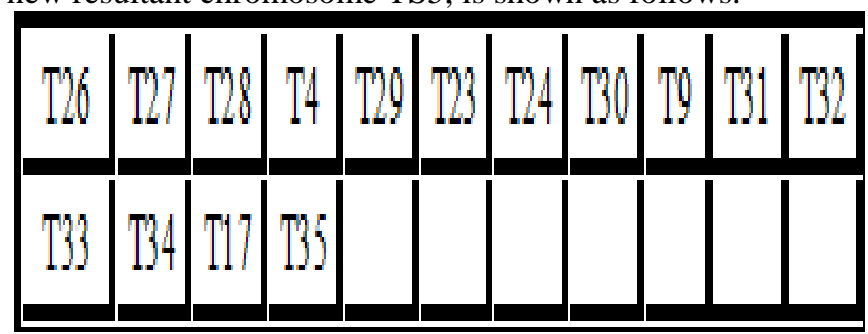

After the second stage crossover and further mutations on genes using the genetic algorithms the resultant analysis of the computed details and results attained for the AVISAR framework are being encapsulated and being shown in Table 1.5. Further Table 1.6 provides Analysis of data and results for the various test suites using the GA.

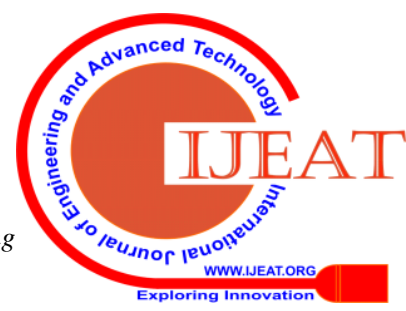




\begin{tabular}{|c||c|c||c||c||c||c|}
\hline Test Suites \& Information & IP-TS & TS1 & TS2 & TS3 & TS4 & TS5 \\
\hline \hline Test Suite Size & 35 & 15 & 15 & 15 & 15 & 15 \\
\hline \# of faults not covered & 0 & 5 & 4 & 5 & 2 & 0 \\
\hline \hline $\begin{array}{c}\text { \# of TC revealing at least a } \\
\text { new fault }\end{array}$ & 12 & 7 & 8 & 7 & 8 & 11 \\
\hline \# of Requirements Covered & 10 & 5 & 7 & 6 & 6 & 10 \\
\hline \hline Are all faults exposed? & Yes & No & No & No & No & Yes \\
\hline METRICS & & & & & & \\
\hline \hline APFD Value (\%) & 66.4 & 81 & 73 & 74 & 70 & 55.9 \\
\hline Requirements Coverage (\%) & 100 & 50 & 70 & 60 & 60 & 100 \\
\hline Fault Coverage (\%) & 100 & 58.3 & 66.7 & 58.3 & 83.3 & 100 \\
\hline \hline Test suite Efficiency (\%) & 34.3 & 46.7 & 53.3 & 46.7 & 53.3 & 73.3 \\
\hline \hline
\end{tabular}

Table 1.5 Summed up results produced by genetic GA for OOP

\section{EXPERIMENTAL SETUP}

For effort estimation during the functioning AVISAR framework we have used the following code block for ensuring the max code coverage.

public GAMaxCodeCoverageCases

( File jF, List $<$ String[] $>$ ea, int mini, int maxi, int sos )

\{ selectedJavaFile $=\mathrm{jF}$;

minIndex = mini;

maxIndex = maxi;

sizeOfSubset $=$ sos; $\}$

public List $<$ Integer $>$ startGA()

int i;

IntegerChromosome ich IntegerGene ig; Integer caseNumber;

Genotype.of( BitChromosome.of(10,0.5) ); final Factory $<$ Genotype $<$ IntegerGene $>>$ gtf $=$

Genotype.of(IntegerChromosome.of( minIndex, maxIndex, sizeOfSubset ));

final Engine $<$ IntegerGene, Integer $>$

engine=Engine.builder(GAMaxCodeCoverageCases::eval,gt f).build();

final Genotype $<$ IntegerGene $>$ result $=$

engine.stream().limit(10).collect(EvolutionResult.toBestGen otype());

System.out.println("Result : \n\t" + result);

System.out.println( "Count of failed cases in Result are: " + String.valueOf(eval(result)) );

List $<$ Integer $>$ subsetCases $=$ new ArrayList $<$ Integer $>()$; ich $=$

result.getChromosome().as(IntegerChromosome.class);

for( $\mathrm{i}=0$; $\mathrm{i}<=$ (ich.length()-1); $\mathrm{i}++$ )

$\{$ ig = ich.getGene(i);

caseNumber = ig.intValue();

subsetCases.add(caseNumber); $\quad$ \}

return subsetCases; \}
excelArray = ea;

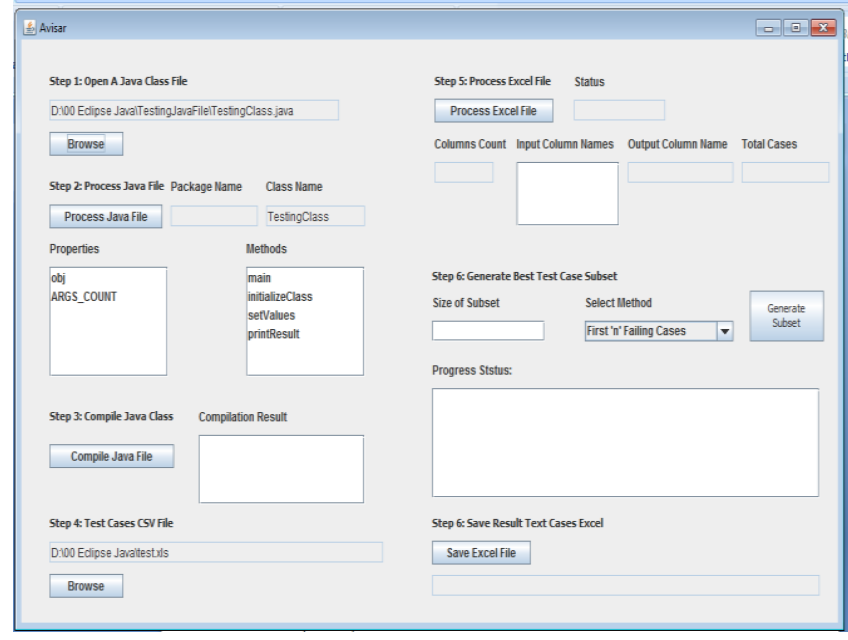

Fig. 5.1. To show the interface of the tool AVISAR for implementation of the proposed work.

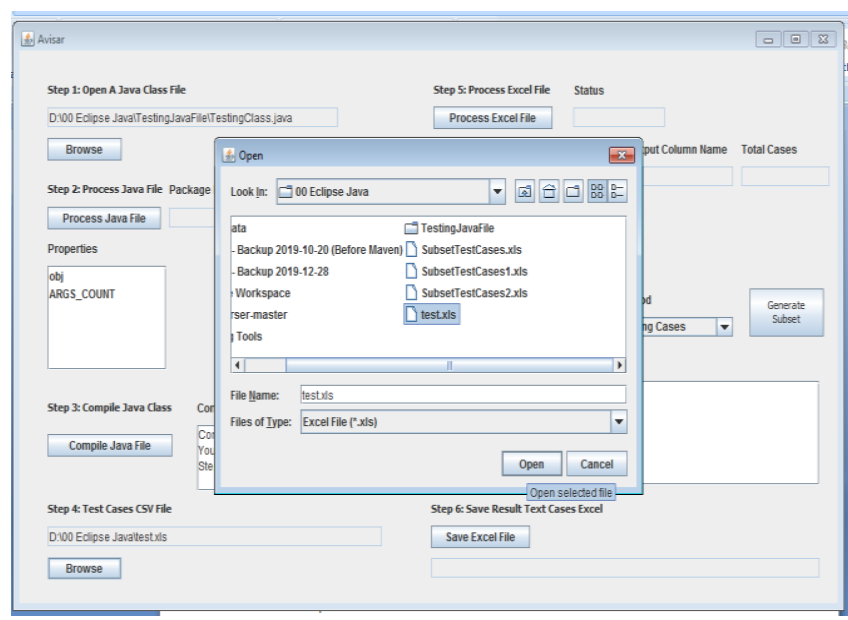

Fig. 5.2. To show the selection of any Object oriented Source code to be tested using AVISAR

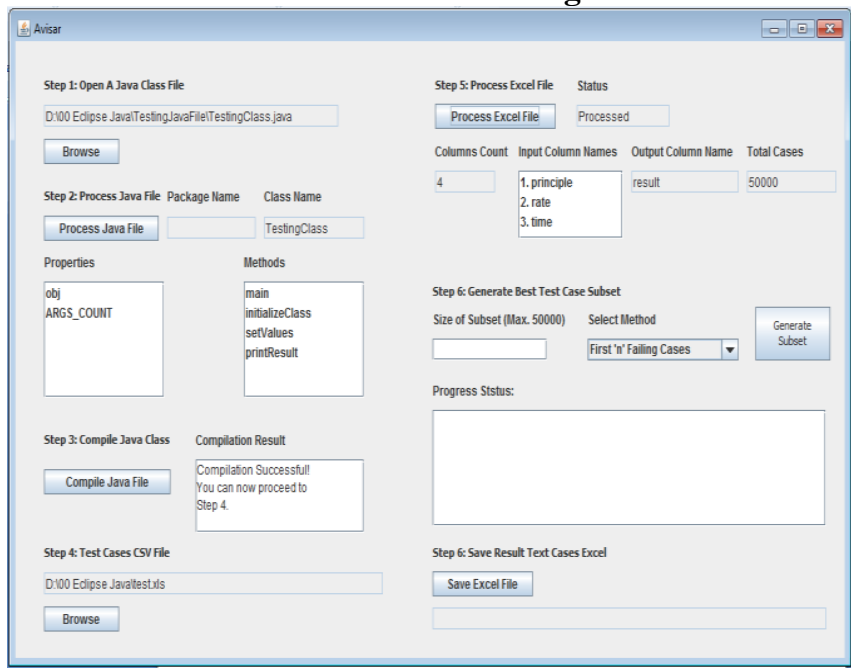

Fig. 5.3.To show the execution of test cases using Genetic Algorithm

Published By:

Blue Eyes Intelligence Engineering \& Sciences Publication

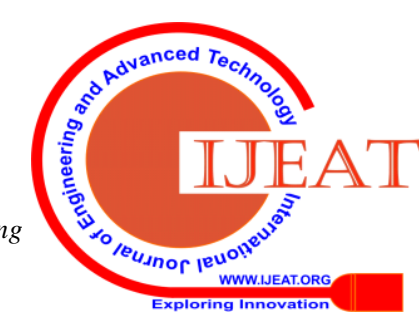




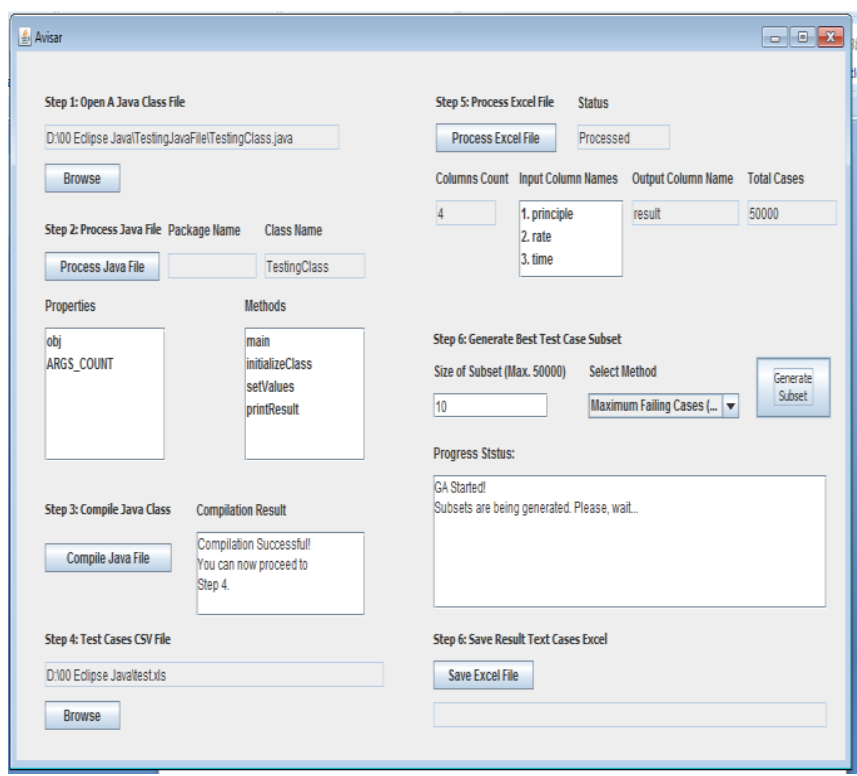

Fig. 5.4. To show the immediate optimized result of test cases using the GA.

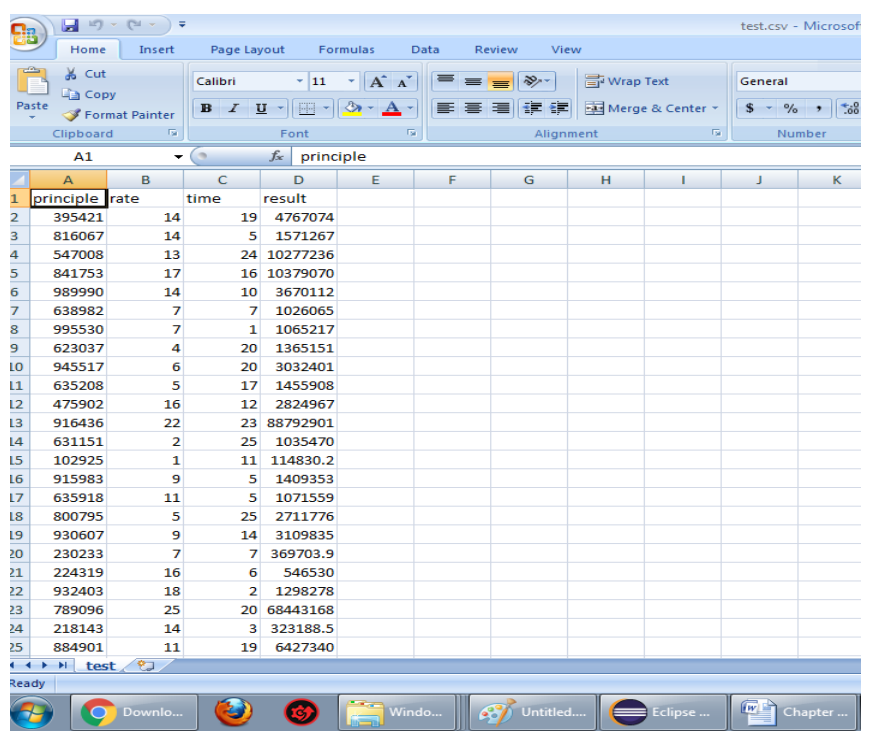

Fig.5.5. To show the Test cases being designed for OOP under Test.

\section{EXPERIMENTAL RESULTS}

When implemented in Java, the proposed OO Testing Framework AVISAR has provided better results for the small chunks of OO codes. We show the results as follows.

6.1 To measure cohesion, we achieved the following graph in Figure 6.1 when it was drawn between a set of functions and attributes together with the arcs in the bipartite graph. It showed impromptu results for smaller code, however as size of the code enlarges, it increases to infinity.

\subsection{To display the performance of Complexity Calculator} To measure the Complexity in Figure 6.2, we obtained the following graph, plotted between Number of classes and Number of methods in individual classes.

6.3 To show the overall performances of OOD estimator To measure the object-oriented design estimator, we plot the graph in Fig. 6.3 for the method visibility, Polymorphism, Inheritance, and Encapsulation for estimation of effort for smaller codes implemented using four modules in Java.

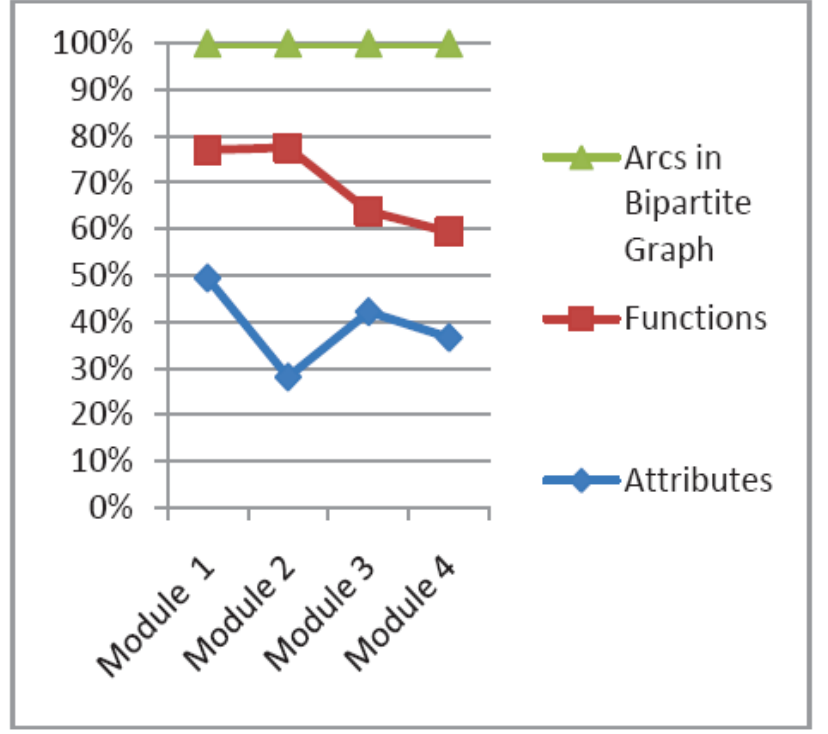

Fig. 6.1 Outturns for the Cohesion Estimator in AVISAR.

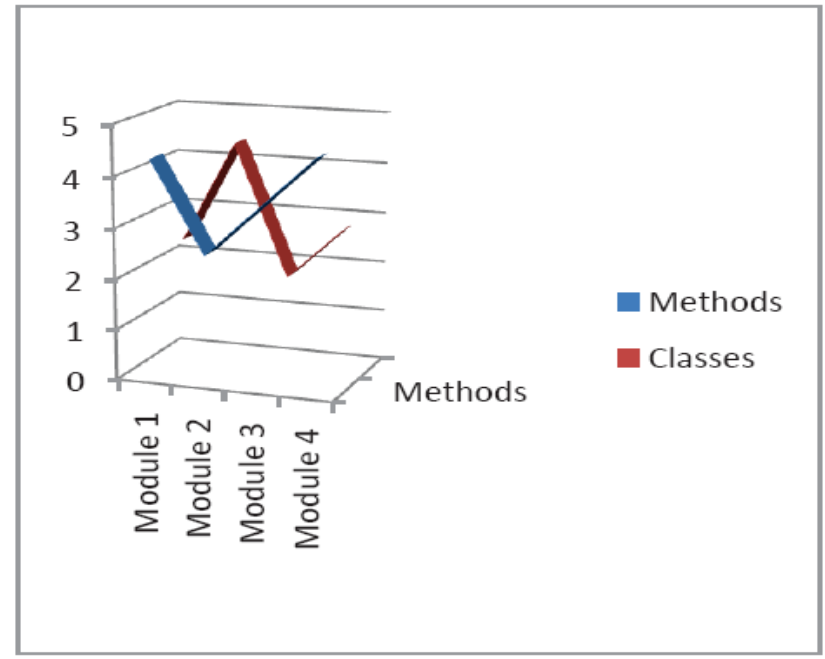

Fig 6.2 Outturns for the of Complexity Calculator in AVISAR.

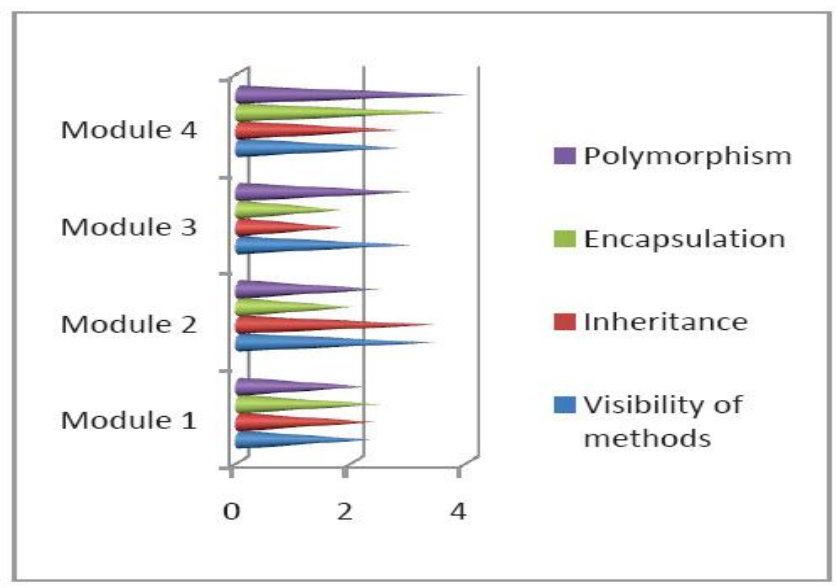

Fig. 6.3 Outturns for the of OOD estimator in AVISAR.

\section{CONCLUSIONS}

Published By:

Blue Eyes Intelligence Engineering

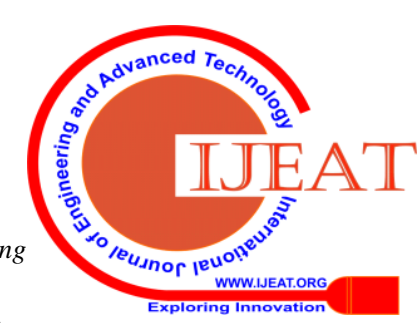


In this research paper, we have proposed methodology for the evaluation of efforts putted in during the carrying out the trails of OOP for the detection of faults, using the AVISAR framework. During the evaluation of efforts using the AVISAR Framework we have observed that the use of GA has proven their worth to provide better results in terms of reduced effort estimation while testing the OOP.

\begin{tabular}{|c|c|c|}
\hline $\begin{array}{l}\text { Test } \\
\text { Suite }\end{array}$ & $\begin{array}{l}\text { Computation } \\
\text { Steps }\end{array}$ & $\begin{array}{l}\text { APFD } \\
\text { Value } \\
(\%)\end{array}$ \\
\hline TS & $\begin{array}{l}{[1(1+3+2+4+7+12+11+17+21+23+26+20) /(12 \times 35)]} \\
+[1 /(2 \times 35)]=1-(147 / 420)+(1 / 70)=1-0.35+0.0143 \\
=0.6643\end{array}$ & 66.43 \\
\hline TS1 & $\begin{array}{l}{\left[1-\left(1+3+2+4+7+12+11+^{*}+^{*}+^{*}+^{*} t^{*}\right) /(12 \times 15)\right]+} \\
{[1 /(2 \times 15)]=1-(40 / 180)+(1 / 30)=1-0.22+0.03=} \\
0.81\end{array}$ & 81.00 \\
\hline TS2 & $\begin{array}{l}{\left[1-\left(3+2+4+7+12+11+14+*+^{*}+*{ }^{*}\right) /(12 \times 15)\right]+} \\
{[1 /(2 \times 15)]=1-(54 / 180)+(1 / 30)=1-0.3+0.03=} \\
0.73\end{array}$ & 73.00 \\
\hline TS3 & $\begin{array}{l}{\left[1-\left({ }^{*}+3+1+4+7+12+11+14+^{*}+*+^{*}+*\right)(12 \times 15)\right]+} \\
{[1 /(2 \times 15)]=1-(52 / 180)+(1 / 30)=1-0.29+0.03=} \\
0.74\end{array}$ & 74.00 \\
\hline TS4 & $\begin{array}{l}{[1-(1+8+2+4+15+12+*+14+3+6+*+1)(12 \times 15)]+} \\
{[1 /(2 \times 15)]=1-(66 / 180)+(1 / 30)=1-0.37+0.03=} \\
0.70\end{array}$ & 70.00 \\
\hline TS5 & $\begin{array}{l}{[1-(3+5+9+4+8+10+5+14+7+6+1+13)(12 \times 15)]+} \\
{[1 /(2 \times 15)]} \\
=1-(85 / 180)+(1 / 30)=1-0.4722+0.03=0.6643\end{array}$ & 55.78 \\
\hline \multicolumn{3}{|c|}{$\begin{array}{l}\text { * refers to the fault not revealed by any of the test cases in the } \\
\text { test suite. }\end{array}$} \\
\hline
\end{tabular}

Table 1.6 Analysis of data and results for the various test suites using the GA.

\section{REFERENCES}

1. Harumi W., Hiroki T., Wenxin Wu , Motoshi Saeki; “A Technique for Analyzing and Testing Object-Oriented Software Using Colored Petri Nets"; IEEE International Conference;1998;Pg.No:182-190.

2. Aynur Abdurazik, Jeff Offutt;" Using Coupling-based Weights for the Class Integration and Test Order Problem" published by Oxford University Press, The British Computer Society, 2006.

3. A.S. Parrish, R.B. Borie, and D.W. Cordes, "Automated Flow Graph Based Testing of Object Oriented Software Modules," Journal of Systems and Software, 23, 1993, pp. 95-109.

4. Jaroenpiboonkit, J. , Suwannasart, T. ;" Finding a Test Order using Object-Oriented Slicing Technique" IEEESoftware Engineering Conference, 2007. APSEC 2007. 14th Asia-Pacific Pg. no: 49-56.

5. R.K. DOONG, P.G. Frankl; "The ASTOOT Approach to Testing Object Oriented Programs"; ACM Transactions on Software Engineering and Methodology, Vol. 3,1994, pages 101-130.

6. Wayne Liu And, Wayne Liu , Paul Dasiewicz;'The Event-Flow Technique for Selecting Test Cases for Object-Oriented Programs";IEEE conference proceedings 1997.

7. D.L. Parnas and Y. Wang, "Simulating the behavior of software modules by trace rewriting systems", IEEE Trans. Software Eng. 20 (10) (1993) Pg.No: 750-759.

8. Ilinca C, Andreas L., Manuel O., Bertrand M.;" ARTOO: Adaptive Random Testing for Object-oriented Software"; published in ICSE'08, May 10-18, 2008, Leipzig, Germany.

9. Yabo Wang, D. L. Parnas;" Simulating the Behavior of Software Modules by Trace Rewriting"; IEEE transactions of software engineering;October1994(Vol.20,No.10) Pg. no.750-759.

10. H.Y. Chen, T.H. Tse, F.T. Chan, and T.Y. Chen., "In black and white: an integrated approach to class-level testing of object-oriented programs." ACM Transactions on Software Engineering and Methodology, 7(3):250-295, 1998.

11. W.K.Chan,T.Y.Chen,T.H.Tse;" An Overview of Integration Testing Techniques for Object-Oriented Programs"; Proceedings of the 2nd ACIS Annual International Conference on Computer and Information Science (ICIS 2002), International Association for Computer and Information Science, Mt. Pleasant, Michigan (2002).

12. Zhe Li, Hamilton M., T.;" An Approach to Integration Testing of Object Oriented Programs"; IEEE transactions, Quality Software, 2007. QSIC '07. Seventh International Conference, Oct. 2007; Pg. No: $268-273$.

13. Alisa Irvine, A. Jefferson Offutt;" The Effectiveness of Category Partition Testing of Object-Oriented Software"; CiteseerX, 1995;

14. Tao Xie, David Notkin;" Automatically Identifying Special and Common Unit Tests Based on Inferred Statistical Algebraic Abstractions"; 2003.

15. S. Savage, M. Burrows, G. Nelson, P. Sobalvarro, T. Anderson; "Eraser A dynamic data race detector for Multithreaded Programs"; ACM transactions on computer systems, 1997.

16. Gerald H., André B., Jan B.; “A distributed real-time java system based on csp"; CiteseerX, 2000.

17. Vats, P., "AVISAR - a three tier architectural framework for the testing of Object Oriented Programs" pub. In Second IEEE International Innovative Applications of Computational Intelligence on Power, Energy and Controls with their Impact on Humanity (CIPECH), 2016.

\section{AUTHORS PROFILE}

Prof. Manju Mandot is a Professor and Director of Directorate of Jan

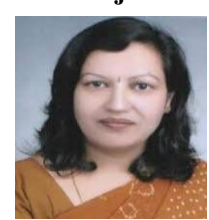
Shikshan and Extension, J.R.N. Rajasthan Vidyapith (Deemed University). She has 27 years of teaching experience. Her research interest includes image processing, E- governance, women empowerment with technology. She is esteemed member of Computer Society of India

Mr. Prashant Vats is working in the field of CSE \& IT as an Assistant

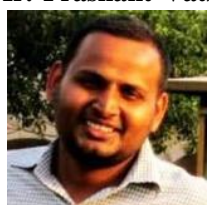
Professor from past 11 years. He has done Diploma in Medical Electronics, B. tech. (IT), M. Tech. (IT) from GGSIPU, New Delhi., MBA \& M.A in Education from IGNOU, New Delhi, PG Diploma in Cyber Laws from Indian Law Institute, New Delhi. He is pursuing Ph.D. in CSE from Banasthali Vidyapith, Rajasthan. $\mathrm{He}$ is a member of IEEE.His research area includes OO paradigm, Software Engineering, IOT, Cloud Computing, BigData. He has published more than 32 research publications in various national \& international journals of repute. 